\title{
A Fresnel Reflection-Based Optical Fiber Sensor System for Remote Refractive Index Measurement Using an OTDR
}

\author{
Jianying YUAN, Chunliu ZHAO*, Manping YE, Juan KANG, \\ Zaixuan ZHANG, and Shangzhong JIN
}

\author{
Institute of Optoelectronic Technology, China Jiliang University, Hangzhou, 310018, China \\ ${ }^{*}$ Corresponding author: Chunliu ZHAO_ E-mail: zhchunliu@hotmail.com
}

\begin{abstract}
In this paper, we propose a Fresnel reflection-based optical fiber sensor system for remote refractive index measurement using the optical time domain reflectometry technique as an interrogation method. The surrounding refractive index from a long distance away can be measured easily by using this sensor system, which operates based on testing the Fresnel reflection intensity from the fiber-sample interface. This system is a simple configuration, which is easy to handle. Experimental results showed that the range of this measurement could reach about $100.8 \mathrm{~km}$, and the refractive index sensitivities were from $38.71 \mathrm{~dB} / \mathrm{RIU}$ to $304.89 \mathrm{~dB} / \mathrm{RIU}$ in the refractive index (RI) range from 1.3486 to 1.4525 .
\end{abstract}

Keywords: Fresnel reflection, optical fiber sensor, remote measurement, OTDR

Citation: Jianying YUAN, Chunliu ZHAO, Manping YE, Juan KANG, Zaixuan ZHANG, and Shangzhong JIN, "A Fresnel Reflection-Based Optical Fiber Sensor System for Remote Refractive Index Measurement Using an OTDR,” Photonic Sensors, 2014, 4(1): 48-52.

\section{Introduction}

Remote monitoring is useful and important for some applications such as chemical industry, marine environments assessment, and pipeline monitoring, and the refractive index (RI) is an inherent characteristic of a substance that is closely related to the physical and chemical properties, hence, a precise detection of the RI is essential in some remote fields [1, 2], for example, the remote monitoring of the refractive index or solution concentration in some dangerous chemical industry is highly necessary for the safety production. Recently, Fresnel reflection-based optic sensors have attracted general interest due to their simple structure, low cost, low cross infection, and durability against the harsh environment [3-5]. In 2013, M. G. Shlyagin et al. [6] proposed an optical fiber refractometer based on the Fresnel reflection in the fiber tip, in which a twin-Bragg grating interferometer is located at some distance from the fiber tip to eliminate the influence of light source fluctuation and environment. Such a structure is complex and not suitable for a remote measurement.

In this paper, we propose a remote refractive index sensor based on Fresnel reflection that can timely tell the RI value from a long distance away, meanwhile, the position of the testing point can be obtained by using a commercial optical time domain reflectometer (OTDR). The theory analysis and experimental setup of the design are introduced and discussed in detail. The experimental setup is simple, which is easy to handle. Experimental results showed that the proposed remote measurement sensor worked well and had a good stability, the sensitivities were from $38.70 \mathrm{~dB} / \mathrm{RIU}$ to

Received: 17 July 2013 / Revised version: 11 October 2013

C The Author(s) 2013. This article is published with open access at Springerlink.com DOI: $10.1007 / \mathrm{s} 13320-013-0131-6$

Article type: Regular 
$304.73 \mathrm{~dB} / \mathrm{RIU}$ in the RI range from 1.3486 to 1.4525 .

\section{Setting and sensing principle}

Figure 1 shows the experimental setup of the proposed remote surrounding refractive index (SRI) sensor. The system includes an optical time domain reflectometer (OTDR) and a long distance single mode fiber whose end is cleaved as a vertical smooth surface which is used as a sensing head. Glycerol solutions with different concentrations are used as the testing samples whose RIs have been calibrated by the Abbe refractometer.



Fig. 1 Experimental setup of the solution concentration sensor.

As shown in Fig. 1, laser light pulses (the wavelength is $1550 \mathrm{~nm}$, and the averaging time is $60 \mathrm{~s}$ ) from the OTDR are emitted into the fiber link, when they reach the interface between the fiber's end surface and sample, Fresnel reflection signals go backward, whose intensities are corresponding to the SRI, then the Fresnel reflection signal and backscattering light signals will be detected by the OTDR (FTB-150), which can be able to resolve intensity changes in backward signals of $0.01 \mathrm{~dB}$. The OTDR system is normally used for the characterization of optical fiber links, it operates by periodically emitting short duration laser pulses into the fiber link, and the backscattering light signal and the Fresnel reflection signal are detected and analyzed by the OTDR computing system. Finally, the backscattering signal and the Fresnel reflection signal levels can be shown on the OTDR trace.

In short-distance measurements, the transmission loss of the optical fiber is so low that won't make much difference to the results, however, a long length optical fiber is used in this remote measurement, thus the transmission loss of the long fiber link cannot be neglected. From different distances, the detected intensity values of reflection signals are not equal, so the intensity of the reflection signal is not suitable to be used as an indicator for measuring the RI. Here, we choose the relative intensity as the indicator [7]:

$$
R=\frac{I_{\text {out }}(\lambda)}{I_{\text {in }}(\lambda)}=\left(\frac{n_{\text {core }}-n_{s}}{n_{\text {core }}+n_{s}}\right)^{2}
$$

where $I_{\text {out }}$ is the intensity of the Fresnel reflection light from the fiber-sample interface, $I_{\text {in }}$ is the input intensity of the laser light, and $n_{\text {core }}$ and $n_{\mathrm{s}}$ are the reflective index (RI) of the fiber core and that of the sample under test, respectively. $n_{\text {core }}$ is a constant (be taken as 1.467 at the room temperature $25{ }^{\circ} \mathrm{C}$ ) which is determined by the physical parameter of the fiber. $R$ in $\mathrm{dB}$ is also the height of the reflection peak on the OTDR trace as marked in Fig. 2. We can measure the surrounding refractive index from a

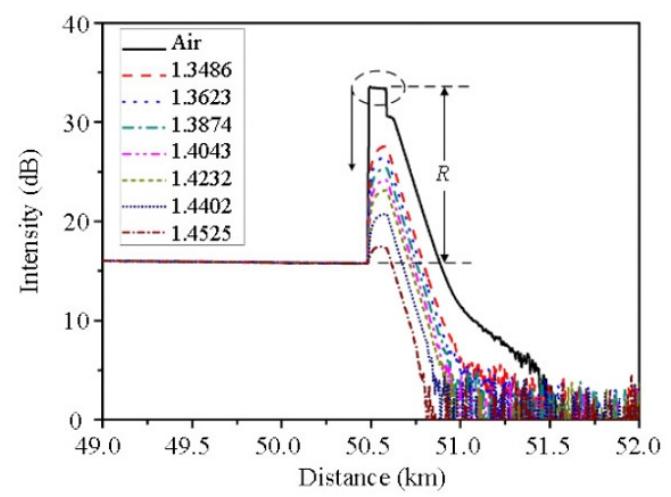

(a)

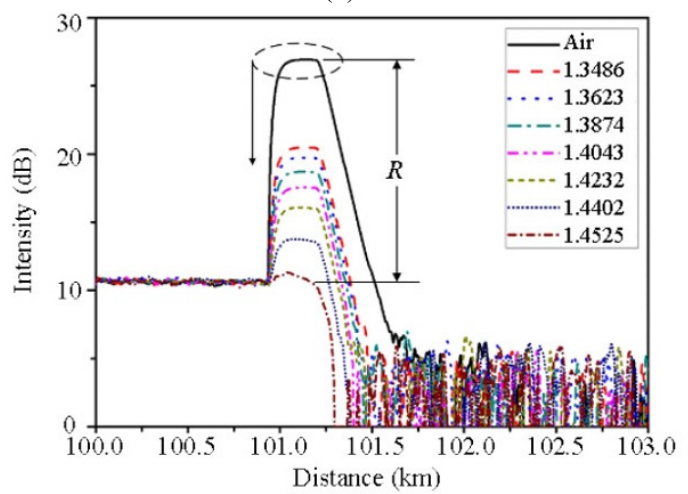

(b)

Fig. 2 Enlarged views of two reflection peaks when sensing heads are located at the distances of (a) $50.4 \mathrm{~km}$ and (b) $100.8 \mathrm{~km}$, respectively. 
measurement of the height value of the reflection peak on the OTDR trace.

\section{Experiment and results}

We utilized this sensor system to make two remote SRI measurements in which the testing solutions were located at $50.4 \mathrm{~km}$ and $100.8 \mathrm{~km}$ away, respectively. The experiments were carried out within two laser pulse widths, the 1- $\mu$ s width [Fig. 2(a)] and 2.5- $\mu$ s width [Fig. 2(b)] for 50.4-km and 100.8-km SRI measurements, respectively.

As shown in Fig. 2, it can be seen that every trace has its clear reflection peak, and the height of each reflection peak decreases monotonically with an increase in the refractive index. Figure 3 shows the relationship between $R$ and $S R I$ for the $50.4-\mathrm{km}$ and 100.8-km $S R I$ measurements. Experimental data show that two existing results are in a good agreement with each other, so that two results can use one curve to fit them. The fitting function is $y=$ $10.124 \times \lg [(1.467-x) /(1.467+x)]+23.771$ with fitting degrees of 0.9993 and 0.9984 for the $50.4-\mathrm{km}$ and $100.8-\mathrm{km}$ SRI measurements, respectively. In the fitting function, $x$ and $y$ are in the corresponding to $S R I$ and $R$ in $\mathrm{dB}$, respectively. We can get the sensitivity through the derivation of the fitting function. The refractive index RI sensitivities for this sensor system were from $38.70 \mathrm{~dB} / \mathrm{RIU}$ to $304.73 \mathrm{~dB} / \mathrm{RIU}$ in the RI range from 1.3486 to 1.4525. The OTDR with a $0.01 \mathrm{~dB}$ measurement precision was used in our experiments, so the

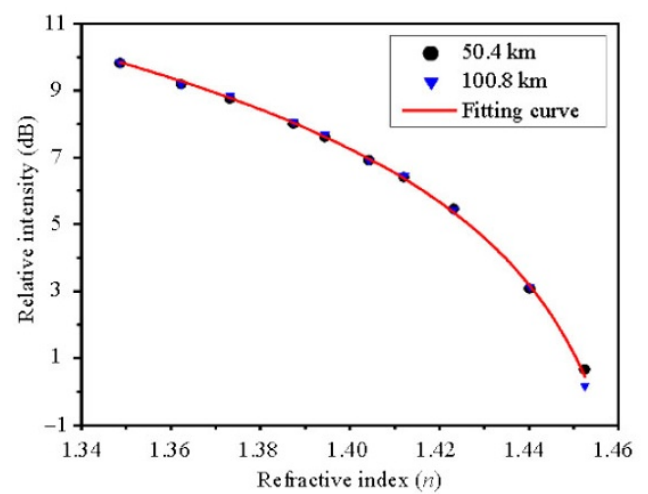

Fig. 3 Relationships between the relative intensity and refractive index for the $50.4-\mathrm{km}$ test and the $100.8-\mathrm{km}$ test. precision of the $R I$ could reach about $2.58 \mathrm{e}-4$ in the RI value of 1.3486 in theory.

Further, the ambient temperature effect on the refractive indices of fiber cores needs to be considered, which will affect the results of the measurement. The thermo-optic coefficient of the optical fiber is about $6.45 \times 10^{-6} /{ }^{\circ} \mathrm{C}$ at the wavelength of $1550 \mathrm{~nm}$, thus the maximum error of $R$ can be calculated according to (1). The maximum error is about $0.045 \mathrm{~dB}$ with the temperature change in the range of $0-100{ }^{\circ} \mathrm{C}$, when the value of $n_{s}$ is assumed as 1.3486. Thus, the resolution of the system is affected by the temperature change and is limited at about $1.0 \mathrm{e}-3$ when the OTDR with the intensity resolution of $0.01 \mathrm{~dB}$ is used in our experiments. But when the proposed sensor works in the temperature change less than $30{ }^{\circ} \mathrm{C}$, the measurement error caused by the temperature effect will decrease to below $1.0 \mathrm{e}-4$ and can be neglected at times.

For remote applications, the attenuation or loss of light caused by fiber-bending, strains and stresses in the fiber link is an important issue, which will affect the results of the measurement. We investigated the system's repeatability and accuracy by repeating the test with a length of the fiber link as $100.8 \mathrm{~km}$, in which the samples' true $R I$ values were 1.0000 and 1.4043 , respectively. In addition, we applied a stress on the position of about $50.4 \mathrm{~km}$ in the fiber link, which caused a loss in the middle of the fiber link. The output OTDR traces of these measurements are given in Fig. 4.

As it can be easily observed from Fig. 4, there is a step loss in the middle position of the dotted lines, which is about $1.4523 \mathrm{~dB}$. After this position, the intensity of light back scattered and the height of the reflection peak for the measurements decrease with certain attenuation, and the differences between two curves are almost equal to the step loss. For these measurements, the measured relative intensities and the calculated RIs are given in Table 1. The calculated $R I \mathrm{~s}$ are obtained through substituting these measured height values into the above 
mentioned fitting function.

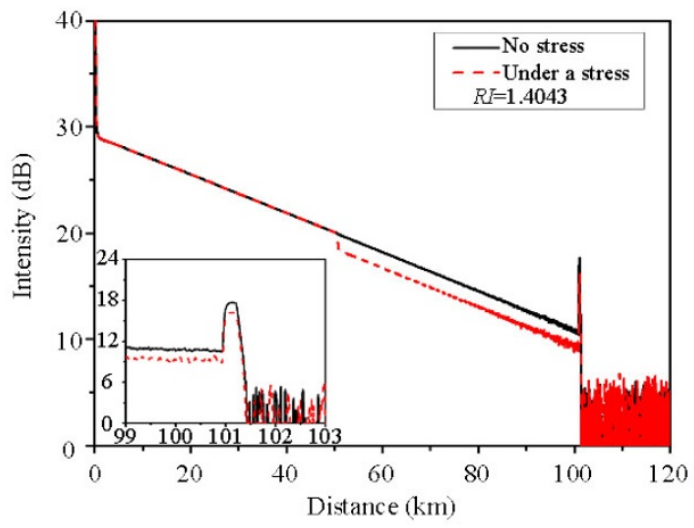

(a)

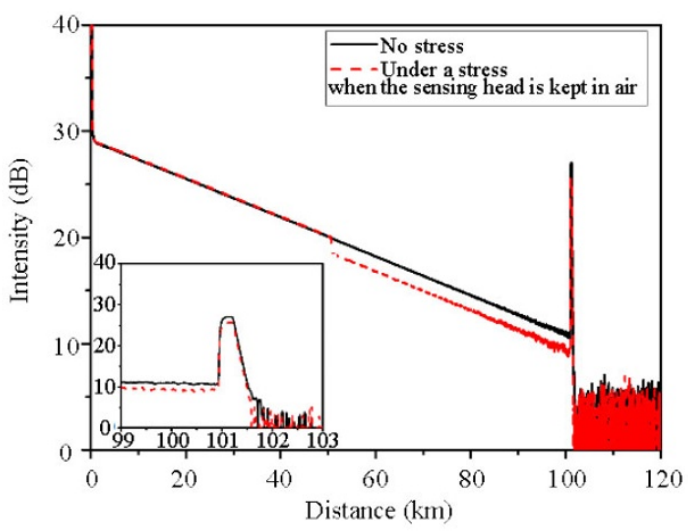

(b)

Fig. 4 Output OTDR traces when sensing heads are inserted in (a) air and (b) a sample whose $R I$ is 1.4043: the insets show the enlarged views of two reflection peaks.

Table 1 Measured relative intensities and the calculated RIs.

\begin{tabular}{c|c|c|c|c}
\hline \multirow{2}{*}{ Samples } & \multicolumn{2}{|c|}{ No stress } & \multicolumn{2}{c}{ Under stress } \\
\cline { 2 - 5 } & Measured $R$ & $\begin{array}{c}\text { Calculated } \\
R I\end{array}$ & $\begin{array}{c}\text { Measured } \\
R\end{array}$ & $\begin{array}{c}\text { Calculated } \\
R I\end{array}$ \\
\hline Air $(n=1.0000)$ & 16.446 & 1.0006 & 16.448 & 1.0004 \\
\hline $\begin{array}{c}\text { Glycerol } \\
(n=1.4043)\end{array}$ & 6.953 & 1.4044 & 6.955 & 1.4043 \\
\hline
\end{tabular}

As Table 1 implies, the measured relative intensity $R$ remains constant with or without a stress in the fiber link for the same sample. By comparing the true values in Table 1, the calculated values have reached the very high accuracy. The maximum error is about $6 \mathrm{e}-4$. This means that the measurement error caused by the bending loss or stress in the fiber link is negligible. It can be also deduced that the laser power fluctuation has almost no effect on the results by using this method, as well as the usage of connectors which can be treated as a loss along the fiber. Hence, the proposed RI remote measurement sensor can realize the remote measurement with a good stability and can be a good candidate for some remote applications.

In this system, the average fiber loss was about $0.186 \mathrm{~dB} / \mathrm{km}$, and the OTDR' dynamic range was about $23 \mathrm{~dB}$ when the measurement range of the OTDR was selected to be $160 \mathrm{~km}$, so the sensing range could reach about $124 \mathrm{~km}$. When the measurement range of the commercial OTDR we used in the system was selected to the maximum $(260 \mathrm{~km})$, the OTDR' dynamic range was about $30 \mathrm{~dB}$, so the maximum sensing distance of the setup could approach about $161 \mathrm{~km}$. The measurement range of the system was determined by the fiber loss, OTDR's dynamic range, spatial resolution, detection sensitivity, and length of the fiber link. Certainly, the system could be improved by using a higher performance commercial optical time domain reflectometer. Moreover, cascading some optical amplifiers can help to achieve a further monitoring [8-10].

\section{Conclusions}

A simple Fresnel reflection-based optic fiber sensor has been proposed for the remote refractive index measurement. It operates based on the Fresnel reflection from the interface between the sensing head and sample and use the optical time domain reflection technique as an interrogation method. Such a spot-measuring method can avoid the cross infection between samples effectively. The proposed sensor system has been proved through our measurements that it has a good stability and can be a good candidate for the remote RI monitoring.

\section{Acknowledgement}

This work was supported by the National Natural Science Foundation of China under Grant No. 61108058, the National Basic Research Program of China (973 program) under Grant No. 
2010CB327804, the National Key Technology R\&D Program 2011BAF06B02, the Science and Technology Commission of Shanghai Municipality of China under Grant No.10595812300, and The Program Funded by Scientific Research and Innovation Team of College Students in Zhejiang Province.

Open Access This article is distributed under the terms of the Creative Commons Attribution License which permits any use, distribution, and reproduction in any medium, provided the original author(s) and source are credited.

\section{References}

[1] H. Minnato, Y. Kakui, A. Nishimoto, and M. Nanjo, "Remote refractive index difference meter for salinity sensor," IEEE Transaction on Instrumentation and Measurement, 1989, 38(2): 608-612.

[2] M. Bravo, J. M. Baptista, J. L. Santos, M. Lopez-Amo, and O. Frazão, "Ultralong $250 \mathrm{~km}$ remote sensor system based on a fiber loop mirror interrogated by an optical time-domain reflectometer," Optics Letters, 2011, 46(20): 4059-4061.

[3] J. R. Zhao, X. G. Huang, W. X. He, and J. H. Chen, "High-resolution and temperature-insensitive fiber optic refractive index sensor based on Fresnel reflection modulated by Fabry-Perot interference," Journal of Lightwave Technology, 2010, 28(19): 2799-2803.

[4] C. L. Zhao, J. H. Li, S. Q. Zhang, Z. X. Zhang, and S. Z. Jin. "A simple Fresnel reflection-based optical fiber sensor for multipoint refractive index measurement using an AWG," IEEE Photonics Technology Letters, 2013, 25(6): 606-608.

[5] J. H. Chen and X. G. Huang, "Fresnel-reflection -based fiber sensor for on -line measurement of ambient temperature," Optics Communications, 2010 , 283(9): 1674-1677.

[6] M. G. Shlyagin, R. M. Manuel, and Ó. Esteban, "Optical-fiber self-referred refractometer based on Fresnel reflection at the fiber tip," Sensors and Actuators B, 2013, 178: 263-269.

[7] M. Born and E. Wolf, Principe of optics, Chapter 1. Beijing: Science Press of China, 1981.

[8] D. C. Dini, J. B. Rosolem, and J. E. Urso, "Active wavelength converter for use with an optical timedomain reflectometer (OTDR) and method for increasing OTDR supervision distance range," US Patent 7369219 B2, May 6, 2008.

[9] H. H. Lee, Y. H. Nam, D. Lee, H. S. Chung, and K. Kim, "Demonstration of a low-cost 1625-nm OTDR monitoring for $350-\mathrm{km}$ WDM networks with semiconductor optical amplifiers," IEEE Photonics Technology Letters, 2005, 17(4): 852-854.

[10] Z. Zhang, "Recent progress in distributed optical fiber Raman photon sensors at China Jiliang university," Photonic Sensors, 2012, 2(2): 127-147. 